\title{
Schema Theorem Based on Probability for Multigenic Chromosomes Genes Expression Programming
}

\author{
Huifang Cheng ${ }^{1 *}$, Jianli Zhao ${ }^{2}$ and Haitao Wang ${ }^{3}$ \\ ${ }^{1}$ School of Information and Electric Engineering, Hebei University of Engineering \\ Handan 056038, P.R. China \\ ${ }^{2}$ Hebei Electric Power Design \& Research Institute, Shijiazhuang, 050031 \\ ${ }^{3}$ College of Science, Hebei University of Engineering, Handan 056038 \\ hfcheng4@163.com
}

\begin{abstract}
Schema theorem is the theoretical foundation of the evolutionary algorithm. This paper focuses on a new, and general schema theory for multigenic chromosomes gene expression programming. The theory is applicable to every operator in GEP via the method of probability. Not only the destruction but also the creation probability of the schema is considered in the evolutionary process. An equivalent form of general schema theory of GEP was deduced. The result shows that the individuals with high fitness values, shorter schema order, more function nodes, and the shorter the insertion sequence have the greater transmission probability than the average ones.
\end{abstract}

Keywords: Gene Expression Programming; GEP Schema Theory, Multigenic Chromosomes

\section{Introduction}

Schema theorem is the theoretical foundation of the evolutionary algorithm. It mainly explains how the population varies dynamically over time in evolutionary process; predicts the number of the instances of the schema in the next generation and provides the theoretical direction for improving the GEP's performance. Binary coded genetic algorithm (GA) as in [1], the tree structure coded genetic programming (GP) as in [2], [3] and linear coded and tree expression of gene expression programming (GEP) as in [4] all belong to evolutionary algorithm. Scholars in order to break through the former limitations did some research, the latter is the result of the study. The three evolutionary algorithms not only have a homologous relation but also have an intercommunity in theory. After some excellent early efforts leading to different GA and GP schema theorems as in [1], [2], [5]-[18], only very recently GEP schema theorem has become available as in [19], and which give just worst-cast-scenario schema theorem for single gene chromosomes GEP. This paper presents a new general schema theory for gene expression programming which is applicable to multigenic chromosomes and all of the standard operators.

The content of the paper is organized as follows. Firstly, the notion of hyperschema theorem is analyzed and some concepts about GEP are included in Sec.2. Then in Sec.3, how these ideas can be used to build probabilistic models of some typical genetic operators were provided. Based on these a new and general schema theory for multigenic chromosomes gene expression programming is proved. Finally, Some conclusions are drawn in Sec.4. 


\section{Gene-hyperschema Theorem and Multigenic Chromosomes GEP Schema}

In gene expression programming, the chromosome consists of a linear, symbolic string of fixed length composed of one or more genes. GEP genes are composed of two different domains - a head and a tail domain - each with different properties and functions. The head domain contains symbols that represent both functions and terminals, whereas the tail is composed of only terminals. For each problem, the length of the head $\mathrm{h}$ is chosen, whereas the length of the tail $t$ is a function of $h$ and the number of arguments of the function with more arguments $\mathrm{n}_{\max }$ (also called maximum arity) and is evaluated by the equation: $\mathrm{t}=\mathrm{h}\left(\mathrm{n}_{\max }-1\right)+1$. For the rest of the related concepts about GEP please refer to [20]. Like all genetic algorithms as in [1,2], gene expression programming works with populations of individuals and, therefore, some kind of initial population must be created in order to get things started. Subsequent populations are descendants, via genetic modification, of this initial or founder population. A new population gives rise to another new population. This process is repeated for a certain number of generations or so until a best solution has been found. The implementation of the algorithm process is as in [20].

\subsection{Gene-hyperschema}

Definition Gene-hyperschema $\mathrm{Hg}$ is a rooted contiguous tree fragment and represents genes having the same shape as $\mathrm{Hg}$. The root node is built using function set and terminal set $F \cup T \cup\{=, \#\}$, where the wildcard ' $=$ ' represents a sure node, and '\#' is interpreted as any valid subtree. For example, if $F=\{+, *\}$ and ${ }^{T}=\{a, b\}$ the gene-hyperschema $(=a \#)$ would represent all the instances of the genes with in the following characteristics: (1) the root node is any of the symbols in the function set or terminal set, if the ' $=$ ' represents any valid node in the terminal set, the length of the ORF is 1 . But if not, (2)'a' is the first argument of the root node; (3) The second argument is any valid subtree.

From the definition of gene-hyperschema, we can see it is a summary of Rosca's variable length schemata theorems and fixed length schemata theorem [12]. If you have a gene-hyperschema theory, you can get overall applicable results for any scale multigenic chromosomes population as below.

\subsection{Multigenic Chromosomes GEP Schema}

Definition: A chromosome schema string linked by several gene-hyperschemas, which is named Multigenic Chromosome GEP Schema' and denoted with an H. For example, an $\mathrm{H}$ contains three gene-hyperschemas that can be described as (\& as the connector, Hgi is the $\mathrm{i}^{\text {th }}$ gene's gene-hyperschema): $H=\{H g 1 \& H g$ \& \& $H g 3\}=\{(+\# a b) \&(+a b \#) \&(+\# b a)\}$.

\section{General Schema Theory for Multigenic Chromosomes Gene Expression Programming}

In 1998 Ricardo Poli come up with ideas that the selection/crossover/mutation process can be seen as a Bernoulli trial ( a newly created individual either samples or does not sample a schema $\mathrm{H}$ ) and, therefore, the number of instances of $\mathrm{H}$ in the next generation, $m(H, t+1)$,is a binomial stochastic variable [12]. If we denote with $\alpha(H, t)$ the probability that a newly created individual samples the schema $\mathrm{H}$, which I term the total transmission probability of $\mathrm{H}$, an general schema theorem expression is simply (1)

$$
E[m(H, t+1)]=M \cdot \alpha(H, t)
$$

Here $E[]$ is the expected number of individuals matching the schema $\mathrm{H}$ within the $(\mathrm{t}+1)^{\mathrm{th}}$ generation, and $\mathrm{M}$ is the number of individuals in the population (population 
scale). In the analysis process, since different genetic operators bring much different results, we aim at using every different genetic operator to formulate different schema theorems. This paper basically introduces eight relevant schema theorems for different genetic operators: Replication and Selection, Two-point mutation of individual, Transposition of IS Elements, Root Transposition, Gene Transposition, One-point Recombination, Two-point Recombination, and Gene recombination.

In gene expression programming, I can know exactly when modifications occur. And all the genetic operators perform in an orderly fashion, starting with replication and continuing with mutation, transposition and recombination. It must be pointed out that the order in which the latter modify the genome is not important to the final outcome. Except for replication, which copies exactly the genomes of all the selected individuals, all the remaining operators randomly pick up the chromosomes to be subjected to a certain modification. And, except for mutation, each operator is not allowed to modify a chromosome more than once. For instance, for a population of 10 individuals, a recombination rate of 0.8 means those eight different chromosomes are randomly chosen to recombine [20].

\subsection{The design of replication and selection operators}

The first step of the selection process is to calculate the fitness function value. Each individual has a choice probability in the selection, The probability of an individual being selected is proportional to the ir fitness as determined by the roulette selection. The total transmission probability of $\mathrm{H}$ is:

$$
\begin{aligned}
& \alpha_{s}^{\prime}(H, t)=p(H, t) \\
& =\frac{\sum_{x \in I(H, t)} f(x)}{\sum_{j=1}^{M} f\left(x_{j}\right)}=\frac{\sum_{x \in I(H, t)} f(x)}{M \bar{f}(t)}
\end{aligned}
$$

Where $\mathrm{p}(\mathrm{H}, \mathrm{t})$ is the probability of selection of the $\mathrm{H}$ within the $\mathrm{t}^{\text {th }}$ generation, $\mathrm{f}(\mathrm{x})$ is the fitness of the individual $\mathrm{x}, \bar{f}(t)$ is the mean fitness of the individuals in the population within the $t^{\text {th }}$ generation, $\mathrm{p}(\mathrm{H}, \mathrm{t})$ is the collection of individuals matching the schema $\mathrm{H}$ within the $\mathrm{t}^{\text {th }}$ generation.

Proof: Only replication and roulette selection are switched on, Thus, it is proved.

Equation (2) indicates transmission probability of schema $\mathrm{H}$ is proportional to the schema fitness and is inversely proportional to the average fitness of the population; In GEP, high fitness schemas will grow rapidly and fill the population, thereby reducing the diversity of groups if only replication and roulettewheel selection are switched on.

\subsection{The Design of Mutation Operator}

Mutation operator which through random change certain genes to introduce new individual chromosomes so that can crease the diversity of population. only mutation is switched on, the total transmission probability of $\mathrm{H}$ is:

$$
\begin{gathered}
\alpha_{m}^{\prime}(H, t)=\left(1-p_{m}\right) \cdot p(H, t)+p_{m} \cdot \alpha_{m}(H, t) \\
\alpha_{m}(H, t)=\left(1-p_{m}\right)^{O(H)}+\prod_{i=1}^{n}\left(1-p_{m}\right)^{N\left(G_{i}\right)-h_{i}-t_{i}} \cdot\left(\frac{p_{m}}{|F|+|T|-1}\right)^{h_{i}} \cdot\left(\frac{p_{m}}{|T|-1}\right)^{t}
\end{gathered}
$$

Where $p_{m}$ is the probability of mutation (per node), ${ }^{N}\left(G_{i}\right)$ is the number of nodes in the $i^{\text {th }}$ gene, $\mathrm{O}(\mathrm{H})$ is the number of non-\# and non-= symbols in the schema $\mathrm{H}$ which $\mathrm{I}$ 
term the order of $\mathrm{H}$. Within an $\mathrm{H}, \mathrm{m}$ refers to the number of nodes in the head part; and $\mathrm{n}$ refers to the number of nodes in the tail part.

Proof: The probability that the individual will not be changed is $1-p_{m}$, and then the probability that the schema is not disrupted (survival) is ${ }^{\left(1-p_{m}\right) O(H)}$. The ith gene become an instance of the ith gene schema Hgi after mutation operation, meanwhile there are ${ }^{h_{i}}$ nodes be changed in the head part and $t_{i}$ nodes in the tail part for an $\mathrm{n}$-genes individual I. In order to maintain the chromosome structure after the mutation operation, any symbol in the head can be turned into a function symbol or terminal symbol. In the tail symbols can only become terminal symbols. Thus, it is proved.

Equation (3) shows that when the population scale, F, T, and mutation rate all are fixed values, the longer the schema order, the smaller the transmission probability.

\subsection{The Design of IS Transposition}

Any sequence in the genome can become an IS element and, therefore, the transposition operator randomly chooses the chromosome, the start and termination points of the IS element, and the target site [20]. Only IS transposition is switched on, the total transmission probability of $\mathrm{H}$ is:

$$
\begin{gathered}
\alpha_{I S}^{\prime}(H, t)=\left(1-p_{I S}\right) \cdot p(H, t)+p_{I S} \cdot \alpha_{I S}(H, t) \\
\alpha_{I S}(H, t) \geq 1-\frac{\mathrm{N}(\mathrm{H}) \mathrm{p}_{\text {Is }}}{n(N(g)-N(I S)+1)(h-1)} \cdot \sum_{I \in P} \delta(I \in B(H, I S))
\end{gathered}
$$

Where $p_{I S}$ is the probability of IS transposition. N(IS) is the number of elements in the insertion sequence(IS). $\mathrm{N}(\mathrm{H})$ is the effective length of GEP schema H. P is the population scale.

Proof: The probability that an individual will not be changed is $1-p_{I S}$, and then the probability that the schema is not disrupted (survival) is $\left(1-p_{l s}\right) \cdot p(H, t) . \mathrm{N}(\mathrm{IS})$ is the number of IS elements, $\mathrm{N}(\mathrm{g})$ is the length of a single gene, $\mathrm{h}$ is the head length of the gene, then each gene may have $N(g)-N(I S)+1$ strings, and have $h-1$ target sites, an ngene chromosome after IS operation may have $N(N(g)-N(I S)+1)(h-1)$ possible individuals. If an obtained progeny samples schema $\mathrm{H}$ after IS translocation operation, the function $G(I, H, I S)=\delta(I \in B(H, I S))$ returns 1 , otherwise it returns 0 . When a selected IS string just fell in the range of schema $\mathrm{H}$, the schema will be destroyed. Consecutive identical characters often exist in a specific individual string, so the probability that the individual will successfully match the schema may be greater than the calculated values after each IS transposition. Thus, it is proved.

Equation (4) shows that the longer the individual string and the shorter the IS string, the higher the transmission probability.

\subsection{The Design of RIS Trans position}

All RIS elements start with a function and, therefore, a point is randomly chosen in the head, and from this point onwards, the gene is scanned until a function is found. This function is the first position of the RIS element. If no functions are found, the operator does nothing [20].Only RIS transposition is switched on, the total transmission probability of $\mathrm{H}$ is:

$$
\alpha_{R I S}^{\prime}(H, t)=\left(1-p_{R I S}\right) \cdot p(H, t)+p_{R I S} \cdot \alpha_{R I S}(H, t)
$$




$$
\alpha_{R I S}(H, t) \geq 1-\sum_{I \in P} \frac{N(H) M_{R I S}}{I\left(N\left(g_{f}\right)\right) N(R I S)} \delta(I \notin B(H, R I S))
$$

Where $p_{R I S}$ is the probability of RIS transposition. $I\left(N\left(g_{f}\right)\right)$ is the number of function symbols in the selected gene of the selected individual. $\mathrm{N}(\mathrm{H})$ is the effective length of GEP schema $H . M$ is the population scale. $B(H, R I S)$ : denote the individual samples schema $\mathrm{H}$ after RIS translocation.

Proof: The probability that the individual will not be changed is $1-p_{R I S}$, and then then probability that the schema is not disrupted (survival) is $\left(1-p_{R s}\right) \cdot p(H, t) . \mathrm{N}(\mathrm{H})$ is the length of schema $\mathrm{H}$. A target gene with $I(N(g)$,$) function nodes, then there are { }^{\prime} \cdot I(N(g)$,$) strings which$ may become selected RIS in a chromosome. Individuals and schema $\mathrm{H}$ are given, if the obtained progeny I samples schema $\mathrm{H}$ after RIS translocation operations, the function $G(I, H, R I S)=\delta(I \in B(H, R I S))$ returns 1 , otherwise it returns 0 . Consecutive identical characters often exist in a specific individual string, so matching probabilities may be greater than the calculated values after each RIS transposition. Thus, it is proved.

\subsection{The Design of Ge ne Transposition}

In gene transposition an entire gene works as a transposon and transposes itself to the beginning of the chromosome. In contrast to the other forms of transposition (IS and RIS transposition), in gene transposition, the transposon (the gene) is deleted at the place of origin. This way, the length of the chromosome is maintained[20]. Only GT transposition is switched on, the total transmission probability of $\mathrm{H}$ is:

$$
\begin{aligned}
& \alpha_{g t}^{\prime}(H, t)=\left(1-p_{g t}\right) \cdot p(H, t)+p_{g t} \alpha_{g t}(H, t) \\
& \alpha_{g t}(H, t)=\prod_{i=1}^{n}\left\lceil\frac{N(H g i+1)}{N(H(I) g i)}\right\rceil
\end{aligned}
$$

Proof: Where ${ }^{p_{s t}}$ is the probability of gene transposition. To maintain the original GEP schema the gene-schema of the transposon must be equal to the first gene-schema of the original individual and the gene-schema ${ }^{H i}{ }^{+1}$ prior to the original position of the transposon should be a subset of the gene-schema ${ }_{B g i}+1$, that is $\{I: H(I) g x=H g 1, H(I) g i \supseteq H g i+1(i=1,2, \cdots, x-1) \wedge H(I)=H\}=H \quad$. To create a new matching individual, the individual, $\mathrm{s}$ schema should meet $\{I: H(I) g x \supseteq H g 1, H(I) g i \supseteq H g i+1(i=1,2, \cdots, n-1) \wedge H(I) \neq H\}=H$, where H(I)gi is the ith gene-schema in the individual I and $\mathrm{H}(\mathrm{I}) \mathrm{gx}$ is the gene schema of the transposon. So the schema $\mathrm{H}$ in a large degree will be destroyed and it is unlikely to produce a new matching individual.

Equation (4) (5) (6) showed that the longer the individual, the more function nodes, the smaller schema length, and the shorter the insertion sequence, the greater the transmission probability of the schema is.

\subsection{The Design of One-Point Recombination}

By recombination we can make use of the individual parent compose new offspring. Only one-point recombination is switched on, the total transmission probability of $\mathrm{H}$ is:

$$
\begin{gathered}
\alpha_{c 1}^{\prime}(H, t)=\left(1-p_{c 1}\right) \cdot p(H, t)+p_{c 1} \cdot \alpha_{c 1}(H, t) \\
\alpha_{c 1}^{\prime}(H, t)=1-\frac{p_{c 1}}{N(I)}\left(\delta(H)-\frac{1-p(H, t)}{M} \cdot \sum_{i=1}^{M} \delta(I 1(H, i))\right)
\end{gathered}
$$


Where $p_{c 1}$ is the probability of one-point recombination. The distance between the first and the last certain node within the valid length of the GEP schema $\mathrm{H}$ named defining length, denoted by $\delta(H)$.

Proof: The probability that the individual will not be changed is $1-p_{c 1}$, and then the probability the schema is not disrupted (survival) is ${ }^{1-p_{c 1} \cdot \frac{\delta(H)}{n \cdot L(g)-1}}$. Supposing the two new individuals obtained from the one-point recombination operation sample the model $\mathrm{H}$, the value of $\delta(I 1(H, i))$ OR $\delta(I 2(H, i))$ is $1,^{\alpha_{c 1}(H, t)=\frac{1-p(H, t)}{M N(I)} \cdot \sum_{i=1}^{M} \delta(I(H, i))}$. Thus, it is proved.

\subsection{The Design of Two-Point Recombination}

Only two-point recombination is switched on, the total transmission probability of $\mathrm{H}$ is:

$$
\begin{gathered}
\alpha_{c 2}^{\prime}(H, t)=\left(1-p_{c 2}\right) \cdot p(H, t)+p_{c 2} \cdot \alpha_{c 2}(H, t) \\
\alpha_{c 2}^{\prime}(H, t)=\left(1-\frac{\delta(H)}{n \cdot L(g)-1}\right)^{2}+\frac{1-p(H, t)}{M N(I)} \cdot \sum_{i=1}^{M} \delta(I(H, i))
\end{gathered}
$$

Proof: In two-point recombination the parent chromosomes are paired side by side and two points are randomly chosen by which both chromosomes are split. The material between the recombination points is then exchanged between the two chromosomes, forming two new daughter chromosomes. Two-point recombination can be regarded as a result of two one-point recombination events [22], because the two one-point recombination operations are independent, the minimum transmission probability of schema $\mathrm{H}$ after the two-point recombination event is $\left(1-\frac{\delta(H)}{n \cdot L(g)-1}\right)^{2}$. One of the two new daughter chromosomes matches to the schema $\mathrm{H}$, the value of $\delta(I 1(H, i) I)$ OR $\delta(I 2(H, i))$ is 1 . Where $p_{c 2}$ is the probability of two-point recombination. Thus, it is proved.

\subsection{The Design of Gene Recombination}

Only gene recombination is switched on, the total transmission probability of $\mathrm{H}$ is:

$$
\begin{aligned}
& \alpha_{c g}^{\prime}(H, t)=\left(1-p_{c g}\right) \cdot p(H, t)+p_{c g} \cdot \alpha_{c g}(H, t) \\
& \alpha_{c g}^{\prime}(H, t)=p_{c g} \cdot \frac{\sum_{i=1}^{n-1}\left(\frac{1}{2^{i}} \cdot 2^{n} \cdot 2^{i}\right)}{(n-1) \cdot 2^{2 n}}=p_{c g} \cdot \frac{n}{(n-1) \cdot 2^{n}}
\end{aligned}
$$

Proof: In the gene recombination entire genes are exchanged between two parent chromosomes, forming two new daughter chromosomes containing genes from both parents. Thus, the gene recombination operator randomly chooses the two parent chromosomes and the gene to be exchanged. Suppose the number of genes of the chromosome is n, then GEP schema is $\{H g 1 \& H g 2 \& \mathrm{~L} \& H g n\}$. The individuals in the population can be divided into $2^{\mathrm{n}}$ kinds of situation to match (incomplete match) with the GEP schema. The two parent chromosomes are randomly chosen and there can be $2^{2 \mathrm{n}}$ possibilities, and there are $n-1$ recombination points. Any positions of the recombination points can form $\left(\frac{1}{2^{i}} \cdot 2^{n} \cdot 2^{\prime}\right)$ matching individuals. Where ${ }^{p_{c g}}$ is the probability of gene recombination. Thus, it is proved. 
Equation (7) (8) (9) showed that if the number of genes in an individual is lower, and the defining length is shorter, the transmission probability of schema is higher.

\subsection{General Schema Theory for Multigenic Chromosomes Gene Expression Programming}

To sum up, consider to replication and selection, mutation, transposition and recombination operation at the same time, the expected value of the number of instances of $\mathrm{H}$ in the next generation is:

$E[m(H, t+1)]=M \cdot \alpha(H, t) \geq M \cdot \alpha_{s}^{\prime} \cdot \alpha_{m}^{\prime} \cdot \alpha_{l s}^{\prime} \cdot \alpha_{R l s}^{\prime} \cdot \alpha_{g t}^{\prime} \cdot \alpha_{c 1}^{\prime} \cdot \alpha_{c 2}^{\prime} \cdot \alpha_{c g}^{\prime}$

The value of the transmission probability can be calculated according to (2) (9).

Proof: The transmission probability of schema $\mathrm{H}$ from the th generation to the $(t+1)^{\text {th }}$ generation is the probability of schema $\mathrm{H}$ to be maintained and produced again during a single step evolution. The genetic operator in the evolutionary process is implemented in order, and the order in which the latter modify the genome is not important to the final outcome. Therefore (10) is available by substituting (1) to (2) - (9).

The theory shows that when roulettewheel selection is selected and multipoint mutation, transposition and recombination are switched on in the GEP, the schema with low order, short defining length and higher average fitness than the population will grow rapidly. The main reason is that the schema with higher fitness has higher probability to be selected to the next generation (2). In addition, if the order is small, the destructive power of mutation on the schema is weak (3). If the schema has less head length and contains less function nodes, the schema is more likely to survive the transposition operation (4) and (5). When every genetic defining length is short, the destructive power of the recombination operation on the schema weakens (7) and (8). This train of thought is provided in improving the quality of the GEP population. It's also the starting point of this paper.

\section{Conclusions and Prospective}

Through the above analysis of GEP chromosomes structure, the concept of multigenic chromsomes GEP-hyperschema and theoretically obtained GEP general schema theory are defined by a series of deductions. The GEP general schema theory formula offers the quantificational assessment methods for the GEP algorithm's own improvements.

The next focus of research is to work out more powerful functional schema theorem, which can not only forecast successful matching probability of the next generation, but also predict much further generations, and can provide a uniform accurate model theorem framework. But so far the schema theorem is dependent on the specific genetic operators.

\section{Acknowledgments}

This research has been made possible through financial support by the Nature Science Foundation of Hebei University of Engineering. In addition, the authors would like to thank all the people that contributed to the research, such as J. XIAO and B. J. LI, in particular, H. T. WANG read several drafts of this manuscript, accompanying the process from the beginning and contributing with valuable discussions and suggestions.

\section{References}

[1] J. Holland, Adaptation in natural and artificial systems. (2nd Ed.) [Online].Available: http://www.openisbn.com/preview/0262581116/ (1992).

[2] J. R. Koza, Genetic Programming: On the programming of computers by natural selection.. (2nd Ed.) [Online]. Available: http://www.doc88.com/p-508348349796.html(1992). 
[3] H. V. Jansen, N. R. Tas and J. W. Berenschot, in Encyclopedia of Nanoscience and Nanotechnology, Edited H. S. Nalwa, American Scientific Publishers, Los Angeles (2004), Vol. 5, pp.163-275.

[4] C. Ferreira, Gene expression programming a new adaptive algorithm for solving problems. Complex Systems, vol.13, no. 2, pp. 87-129, Feb. (2001)

[5] Neubauer, The circular schema theorem for genetic algorithms and two-point crossover, in Proc. ICOGAES-IA. London, (1997), pp. 209-214. UK

[6] W. M.Spears, K A. De Jong, "A formal analysis of the role of multi-point crossover in genetic algorithms," Annals of Mathematics and Artificial Intelligence, vol.5, no. 1, pp. 1 26, Jan. (1992)

[7] D. E. Goldberg, K.Sastry, A practical schema theorem for genetic algorithm design and tuning, in Proc. GECCO, San Francisco, CA,( 2001), pp. 328-335. USA

[8] D. W. Gong, X. Y. Sun, Based on the schema theorem of genetic algorithm crossover and mutation probability limit, control and decision, vol. 19, no. 5, pp. 554-556, May, (2004)

[9] H. J. Yang, M. Q. Li, Evolutionary computation in schema theorem and building blocks, Journal of computers, vol. 26, no. 11, pp. 1550-1554, Nov. (2003)

[10] W. B. Langdon, R. Poli, Foundation of Genetic Programming . (2nd Ed.) [Online]. http://books.google.com.hk/books?id=PZli0s95Jd0C\&printsec=frontcover\&dq=Foundation+of + Genetic +Programmin g\&hl=zh-CN\&sa=X\&ei=wKkUUcunKM mZiAfL0IHgCA\&ved=0CDEQ6AEwAA( 2002, Mar 10)

[11] Reilly, An Analysis of Genetic Programming, Ph.D. dissertation, Dept. Ottawa Carleton Institute for Computer Science, Carleton Univ., Ottawa, Canada, (1995). pp.53-59.

[12] R. Poli, W. B. Langdon, Schema Theory for Genetic Programming with One-point Crossover and Point Mutation, Evolutionary Computation, vol. 6, no. 3, pp. 231-252, Mar.(1998)

[13] R. Poli, N. F. McPhee, Exact Schema Theorems for GP with One-point and Standard Crossover Operating on Linear Structures and Their Application to the Study of the Evolution of Size, Proceedings of EuroGP, LNCS, Milan, Springer-Verlag,( 2001). Italy

[14] R. Poli, N. F.McPhee, Exact GP Schema Theory for Headless Chicken Crossover and Subtree Mutation, Proceedings of the 2001 Congress on Evolutionary Computation (2001), Seoul, 2001: 1062-1069. Korea,

[15] R. Poli, N. F. McPhe, Exact Schema Theory for GP and Variable-length GAs with Homologous Crossover, Proceedings of the Genetic and Evolutionary Computation Conference (GECCO-2001), Morgan Kaufmann, San Francisco, Califomia, 2001:104-111.USA

[16] J. P. Rogca, Hierarchical Learning with Procedural Abstraction Mechanisms, Ph.D. dissertation, Dept. Computer Science, Rochester Univ., NY 14627, USA, (1997).

[17] P. A.Whigham, A Schema Theorem for Context-free Grammars, IEEE Conference on Evolutionary Computation, Perth, IEEE Press, (1995), 1:178-181. Australia

[18] Y. C. Zhou, Q. Tong, K. C. Wu, B. P. Yan, Research Progress of Genetic Programming Schema Theorems, Computer Engineering, vol. 29, no. 12, pp. 143-145, Dec. (2002)

[19] Y. Wang, C. J. Tang, N. Yang, Y. Chen, K. K. Xu, The schema theorem of evolution based on gene expression programming, Journal of Sichuan University (Engineering science edition), vol. 41, no. 2, pp. 167-172, Feb. (2009)

[20] C. Ferreira, Gene Expression Programming Mathematical modeling by an artificial intelligence. (2nd Ed.) [Online]. http://www.doc88.com/p-615600243619.html (2006, July 20).

[21] R. Poli, Exact Schema Theory for Genetic Programming and Variable-length Genetic Algorithms with one-Point Crossover, Genetic Programming and Evolvable Machines, no. 2, pp. 123-163, Feb.( 2001)

[22] H. J. Yang, M. Q. Li, Schema Theorem and the Building Block of the Evolutionary Algorithm, Chinese Journal of Computers, vol. 26, no. 11, pp. 1550-1554, Nov. (2003)

\section{Author}

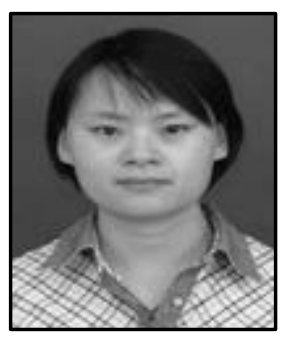

Huifang Cheng, was born in WeiZhuang Village, XingTai City, in 1980. She received the B.S. in Hebei Normal University, ShiJiaZhuang, in 2002, and M.S. degrees in computer application technology from Hebei University of Engineering, Handan, China, in 2009.

From 2002 to 2007, she was an Assistant with information and electricity-engineering institute, Hebei University of Engineering. Since 2007, he has been a lecturer with information and electricityengineering institute, Hebei University of Engineering. She is the author of three books, more than 15 articles. Her research interests include evolutionary algorithm and data mining and applications. 